\section{CA1 triggers the trace}

According to a popular theory, memories are encoded and temporarily stored in the hippocampus, and eventually transferred to the cortex. Indeed, many lesion studies suggest that the hippocampus is not involved in the retrieval of remote memories. However, Goshen et al. now show, using optogenetic inactivation of hippocampal CA1 neurons, that the hippocampus has a role in recall of remote memory, but that in the absence of CA1 activation memories can be retrieved through direct activation of neocortical areas.

The authors optogenetically inactivated glutamatergic (excitatory) CA1 neurons in mice during a contextual fear conditioning test - a hippocampus-dependent memory task. Silencing of these neurons, either during fear conditioning or during a recall test $24 \mathrm{~h}$ later, prevented retrieval of the fear memory (which is expressed as fear behaviour), indicating that CA1 neurons are involved in the formation and recall of recent contextual fear memory.

Surprisingly, inactivation of CA1 neurons during remote recall - up to 12 weeks after fear conditioning - also blocked retrieval of the fear memory. This effect was reversible, as fear memory was restored when the animals were tested the next day without inactivation of CA1 neurons. Strikingly, mice that were tested 5 weeks after training initially showed normal memory retrieval, but when CA1 neurons were inactivated halfway through the recall test, fear behaviour disappeared. These data suggest that the hippocampus is persistently involved in memory retrieval.

In previous studies, genetic, pharmacological or physical lesions of the hippocampus had been performed minutes to weeks before fear memory testing and had been found to affect recent memory but not remote memory. To allow a better comparison with earlier studies, the authors extended optogenetic inactivation from $30 \mathrm{~min}$ before, to the end of the recall test. Consistent with findings from lesion studies, such prolonged inactivation of CA1 neurons blocked retrieval of recent but not remote fear memory.

Studies of FOS expression (a measure of neuronal activation) suggested that CA1 activation during recall may recruit the anterior cingulate cortex (ACC). Accordingly, both real-time and prolonged optogenetic inactivation of ACC neurons during recall impaired remote memory but had no effect on recent memory recall.

These findings indicate that longterm memory retrieval normally involves the hippocampus, possibly as a 'default activator' of the memory trace in neocortical areas. However, this system seems to be flexible in that the memory can also be retrieved directly from the neocortex.

Leonie Welberg

ORIGINAL RESEARCH PAPER Goshen, l. et al.

Dynamics of retrieval strategies for remote

memories. Cell 147, 678-689 (2011)

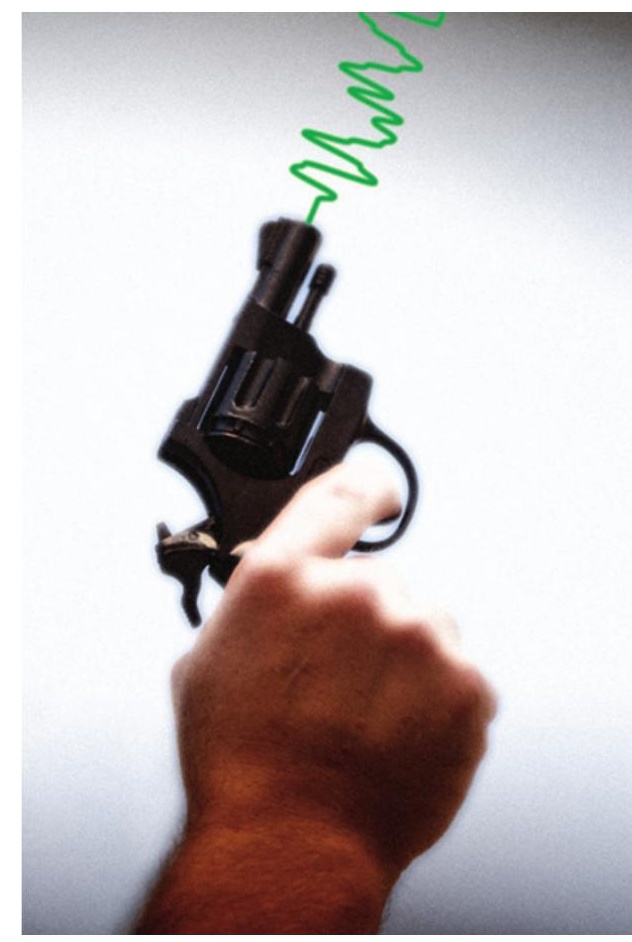

